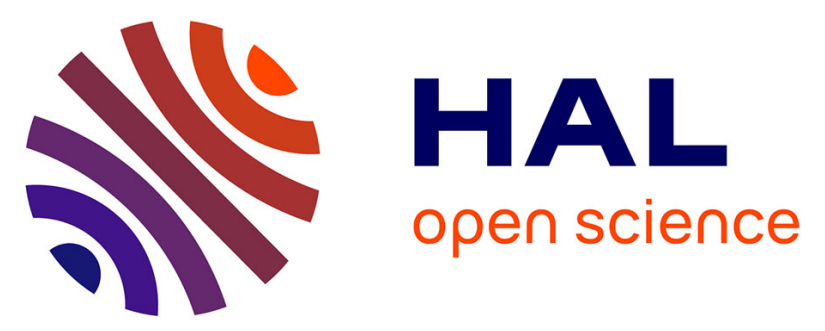

\title{
Terahetz detection by heterostructed InAs/InSb nanowire based field effect transistors
}

A. Pitanti, D. Coquillat, D. Ercolani, L. Sorba, F. Teppe, W. Knap, G. de Simoni, F. Beltram, A. Tredicucci, M. S. Vitiello

\section{- To cite this version:}

A. Pitanti, D. Coquillat, D. Ercolani, L. Sorba, F. Teppe, et al.. Terahetz detection by heterostructed InAs/InSb nanowire based field effect transistors. Applied Physics Letters, 2012, 101, pp.1103. 10.1063/1.4757005 . hal-00749130

\section{HAL Id: hal-00749130 \\ https://hal.science/hal-00749130}

Submitted on 6 Nov 2012

HAL is a multi-disciplinary open access archive for the deposit and dissemination of scientific research documents, whether they are published or not. The documents may come from teaching and research institutions in France or abroad, or from public or private research centers.
L'archive ouverte pluridisciplinaire HAL, est destinée au dépôt et à la diffusion de documents scientifiques de niveau recherche, publiés ou non, émanant des établissements d'enseignement et de recherche français ou étrangers, des laboratoires publics ou privés. 


\title{
THz detection in InAs/InSb nanowire based FET diodes
}

\author{
A. Pitanti, ${ }^{1}$ D. Coquillat, ${ }^{2}$ D. Ercolani, ${ }^{1}$ L. Sorba, ${ }^{1}$ F. Teppe, ${ }^{2}$ W. Knap, ${ }^{2}$ G. De Simoni, ${ }^{3}$ F. Beltram, ${ }^{1,3}$ A. \\ Tredicucci, $^{1}$ and M. S. Vitiello ${ }^{1}$ \\ 1) NEST, Scuola Normale Superiore and Istituto Nanoscienze - CNR, piazza San Silvestro 12, \\ 56127 Pisa - Italy \\ ${ }^{2)}$ Université Montpellier 2 and CNRS, TERALAB-GIS, L2C UMR 5221, 34095 Montpellier, \\ France \\ 3) Center for Nanotechnology Innovation @NEST, Istituto Italiano di Tecnologia, piazza San Silvestro 12, \\ 56127 Pisa - Italy
}

Heterostructured InAs/InSb nanowire based field effect transistors (FET) have been fabricated and tested as $\mathrm{THz}$ radiation detectors. While responsivity and noise equivalent power compare with the ones of InAs nanowire detectors, the presence of small-gap InSb semiconductor gives rise to interesting physical effects such an increase of the detected signal with charge injection through the wire, at odds with standard FETdetectors. Additionally, the photodetected signal voltage changes its sign after a threshold gate bias, which we explain considering surface-related transport and field asymmetries imposed by the use of a lateral gate electrode.

The fast and reliable detection of radiation at $\mathrm{THz}$ frequencies represents an important issue for a full exploitation of $\mathrm{THz}$ technology. Very recently, $\mathrm{THz}$ detection in InAs nanowire (Nw) field-effect transistors (FET) has been reported ${ }^{1,2}$. Due to their reduced size, these systems offer a very large cut-off frequency together with the possibility to be arranged in ordered arrays for imaging applications. Interestingly, the use of heterostructured nanowire based FETs can reveal notewhorty characteristics, since semiconductors with different properties can be used and effects related to charge transport across the heterojunction explored. Recently, InSb nanowires have been widely investigated ${ }^{3,4}$, with the motivation stemming from the small electronic gap, high mobility and easy formation of ohmic contacts which are characteristics of bulk InSb material ${ }^{5}$. These nanowires can be grown with high crystallographic quality on underlying InAs segments, as recently demonstrated ${ }^{6,7}$, and therefore constitute an appealing system for the realization of nanowire based electrical devices. Moreover, when axial charge transport through the heterojunction is investigated a rectification effect due to band lineup can be observed, allowing the realization of axial electrical diodes ${ }^{3}$.

In this Letter, we report on the development of InAs/InSb nanowire lateral gate FETs working as radiation detectors in the sub- $\mathrm{THz}$ frequency range. At odds with standard FET detectors, the detected signal increases when the gate bias is far from the pinch-off voltage and abrubtly changes sign when a certain gate threshold value is reached. We attribute this peculiar behavior to the role of InSb nanowire segments, since standard InAs nanowire FETs behave in analogy to the planar devices ${ }^{1}$. The possibility that the detector signal improves when charge is injected (and not depleted as in usual detector FETs) has been explained through the use of numerical simulations in low-doped nanowire systems, considering the current nonlinearities due to the injection mechanism ${ }^{8}$. Interestingly, this model finds here its first experimental demonstration. On the other hand, the

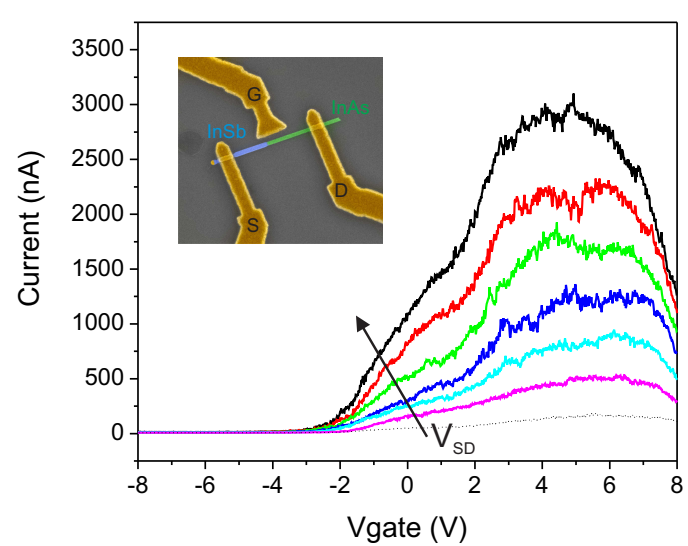

FIG. 1. $\mathrm{I}_{S D}-\mathrm{V}_{\text {gate }}$ at diffent source-drain voltages, from 10 to $60 \mathrm{mV}$. In the inset a SEM image of the contacted wire is shown. The two arms of the log-periodic antenna realized through e-beam lithography are respectively connected to source $(\mathrm{S})$ and gate $(\mathrm{G})$ electrodes, while the current is measured through the drain (D) one.

fact that the responsivity changes its sign is a novel unexpected property, for which we propose a simple explanation based on the gate geometrical asymmetry of our devices. The nanowire samples were grown using the chemical beam epitaxy (CBE) technique ${ }^{7}$ without any intentional doping. Subsequently the devices were fabricated employing e-beam lithography and $\mathrm{Ti} / \mathrm{Au}$ evaporation ${ }^{3}$. To ensure an ohmic contact with the nanowire surface, the latter was properly passivated employing a nonstoichiometric $\left(\mathrm{NH}_{4}\right)_{2} \mathrm{~S}_{x}$ solution $^{9}$. A gate electrode is located on one side of the $\mathrm{Nw}$ (lateral gate) and acts on both InAs and InSb materials on the two sides of the heterojunction. The Source $(\mathrm{S})$ and Gate $(\mathrm{G})$ electrodes were connected to two arms of a log-periodic antenna, whose dimensions are matched with the frequency of the electromagnetic source used for detection experiments ( 0.3 THz), while the Drain (D) contact can be used 
to read out the channel current $I_{D}$ when measuring conductivity, or the output signal $\left(V_{S D}\right)$ when $\mathrm{S}$ is grounded. For transport characterization, a DC bias can be applied to $\mathrm{S}$ and/or $\mathrm{G}$ electrode using standard DAC sources. A SEM image of the final contacted nanowire is shown in the inset of Fig. 1. The $400 \mathrm{~nm}$ wide gate electrode is physically separated from the Nw by a gap of about 75 $\mathrm{nm}$ and properly addresses both InAs and InSb segments. Fig. 1 reports the room-temperature DC charge transport characterization of the device. A small bias is applied to the $\mathrm{S}$ contact (ranging from 10 to $60 \mathrm{mV}$ ), while the $\mathrm{G}$ electrode is swept from -8 to $8 \mathrm{~V}$, clearly modifying the Nw conductivity. When a positive bias is applied to the source contact (InSb side), the InAs/InSb diode is directly polarized, favouring the current flow towards the D contact. Even if nominally undoped, the pinning of the Fermi level at the nanowire surface allows for nonzero conductivity at zero gate bias. By increasing the gate bias, the conductivity grows, showing a majority of electronic carriers with respect to the holes, as expected in similar systems ${ }^{4}$. Even if a saturation of the current would not be surprising, due to quantum capacitance related effects ${ }^{11}$, the conductivity reduction at large gate biases is quite unexpected. Several factors can indeed play a role in such experimental observation: i) gate current leakage, ii) gate field dishomogeneity or iii) a sudden modification of the kind of majority charge carriers. Interestingly, the detection experiments are compatible with the measured charge transport curves. The 0.292 $\mathrm{THz}$ source signal has been shined directly on the sample through parabolic mirrors, reaching a beam spot area of $12.6 \times 10^{-6} \mathrm{~m}^{2}$ with an incident power of $2.1 \mathrm{~mW}$. The detected DC signal (video signal) is measured through a voltage preamplifier (with a gain factor of 25) coupled to a lock-in system ${ }^{1}$. No DC bias has been applied to the $\mathrm{S}$ contact, in such a way that the only voltage bias is given by the electromagnetic source. On the other hand, the device working point has been swept by adding a DC source signal to the $\mathrm{G}$ contact, which sums-up with the AC signal directly coming from the impinging radiation. The video signal for a typical measurement is reported in Fig. 2. A maximum detector responsivity of $1.5 \mathrm{~V} / \mathrm{W}$ has been found, comparable to the one reported for InAs Nw FET detectors ${ }^{1}$; even a rough estimate of the NoiseEquivalent Power (NEP) leads to values similar to the one found in those devices $\left(\sim 1 \times 10^{-8} \mathrm{~W} / \sqrt{\mathrm{Hz}}\right)$. Yet, at variance with InAs nanowire FETs, for gate biases larger than the pinch-off threshold voltage, the video signal increases, at odds also with what happens in classical $\mathrm{FET} /$ diode detectors where the maximum responsivity is found around the channel closure. Most importantly, the video signal changes sign at gate biases larger than $2 \mathrm{~V}$; as can be clearly seen in the inset of Fig. 2, the signal phase changes abruptly of about $180^{\circ}$, accordingly. Indeed, this behavior could be expected by considering the device transport characteristics, and, in particular, the negative differential transconductance found at large gate biases. The responsivity can be extracted from charge

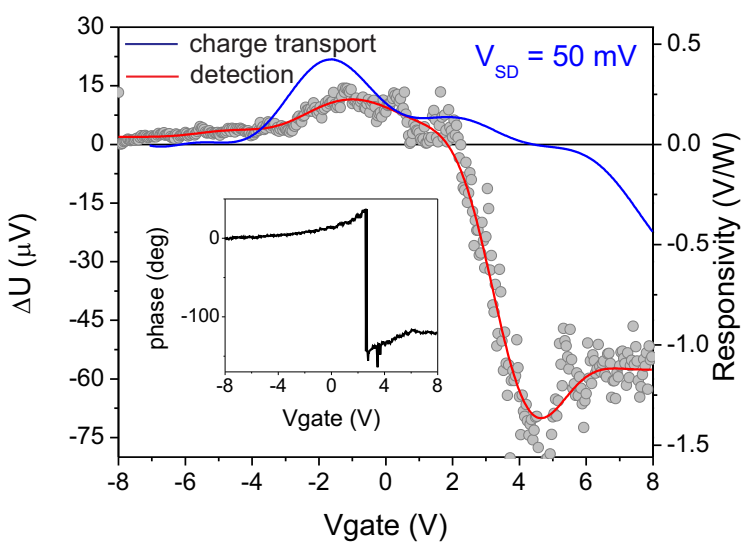

FIG. 2. Video signal obtained in the detection experiment (scatter points, red curve is an interpolation to the data) compared with the responsivity extracted from the transfer characteristics of Fig. 1 (blue curve). Inset: lock-in phase signal recorded during the measurement. Note the $180^{\circ}$ abrupt phase change obtained when the video signal changes its sign.

transport according to the formula ${ }^{12}$ :

$$
R \propto \frac{\Delta U}{1+R_{C H} / Z_{L}} \propto \frac{1}{1+R_{C H} / Z_{L}} \frac{1}{\sigma} \frac{d \sigma}{d V_{\text {gate }}},
$$

where $R_{C H}$ is the channel resistance, $Z_{L}$ the complex load impedance and $\sigma$ the nanowire conductivity. The relevance of the load impedance is well confirmed by the experimental evidence that the video signal phase is not constant when the gate voltage ranges between -8 and $2 \mathrm{~V}$, yet it is slowly drifting, due to load impedance weights ${ }^{13}$. By looking at the magnitude of the video signal, an expected $\mathrm{V}_{S D}$ bias of $50 \mathrm{mV}$ due to the antenna feeding can be readily extracted; therefore, we have applied Eq. (1) to the curve of Fig. 1 corresponding to such a value of $\mathrm{V}_{S D}{ }^{12}$.

The results, plotted in Fig. 2, have been directly compared with the detection curve. Despite difficulties related to the numerical derivative, which makes any direct comparison between the two curves problematic, a reasonably similar trend is observed, with a small peak close to the pinch-off voltage and a signal sign change at about $2 \mathrm{~V}(4 \mathrm{~V})$ for the experimental (computed) curve. It is worth discussing, at least qualitatively, the possible physical mechanisms behind the sign switch in Fig. 2. The gate leakage effect has been discarded by measuring the drain current at zero applied $\mathrm{V}_{S D}$ (reported as a dotted line in Fig. 1). This current intensity has been found to be at least one order of magnitude smaller than the one measured at $50 \mathrm{mV}$ for the same gate field; therefore, it is unlikely to determine such a large modification in the Nw conductivity as the one observed in Fig. 1. The presence of a consistent portion of minority carriers, though reasonable in the proximity of the InAs/InSb junction, is hardly explained at very large $\mathrm{V}_{\text {gate }}$, considering that the whole junction region is influenced by the 

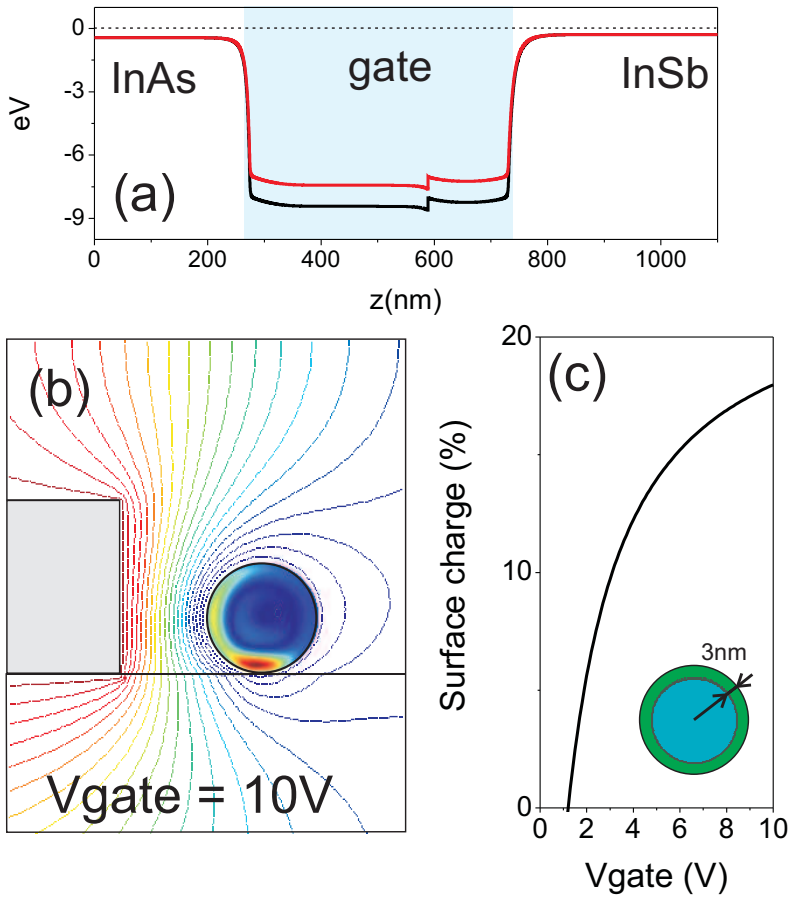

FIG. 3. (a): Schematic of the 1D band line-up of the InAs/InSb FET for a 10V gate bias (Schrödinger-Poisson). The zone in which the gate is expected to have more effect is highlighted; note that it extends very far from the junction region. (b): Charge density and isopotential lines simulated, for a 2D Poisson toy-model, for an applied gate bias of 10 V. (c): The percent of charge localized in the $3 \mathrm{~nm}$-outermost nanowire shell as a function of the lateral gate bias.

lateral gate and thus the energy bands rigidly shift with the gate field. The possible p-type region, which could be present in proximity of the junction, is then washed away in the gate potential obtained at positive applied biases, as schematically reported in Fig. 3 (a). A more plausible explanation can be found in the asymmetries of the electrical gating field induced by the presence of a single side electrode. This asymmetry can indeed lead to strong modification of the charge distribution within the $\mathrm{Nw}$, when large electric fields are applied. This has been recently shown in the symmetry breaking of the levels of a $\mathrm{InP} / \mathrm{InAs} / \mathrm{InP} \mathrm{Nw}$ quantum $\operatorname{dot}^{14}$. Such mechanism is present in our devices too, pushing/pulling the majority carriers towards the nanowire outer surface. It is well known that surface conductance states can be very important in $\mathrm{Nw}$ systems, offering a rich and complicate physics ${ }^{15,16}$. Particularly relevant can be the presence of surface trap states, which can be detrimental to the $\mathrm{Nw}$ conductivity. Then, if a lot of charge is pushed towards the $\mathrm{Nw}$ surface, a conductivity reduction can be expected, similarly to what observed in our experimental data. From a 2D self-consistent toy-model based on the Poisson equation, it is possible to appreciate this kind of effect (more details on the model are discussed in a previous report ${ }^{8}$ ). In fact, as reported in Fig. 3 (b) for very large gate field $\left(\mathrm{V}_{\text {gate }}=10 \mathrm{~V}\right)$, most of the nanowire charge is squezeed towards its lateral edge, due to the strong asymmetry of the electric field. This is indeed expected to strongly influence the charge transport properties of the system. To estimate the magnitude of this effect, we have integrated the charge density over the outermost $3 \mathrm{~nm}$ shell of the nanowire, normalizing it to the charge integrated over the whole $\mathrm{Nw}$ cross-section. The results are reported in Fig. 3 (c), which clearly shows that almost $20 \%$ of the total charge is localized within the surface region for the largest applied biases. Interestingly, the proposed effect is particularly relevant when InSb nanowires are investigated, and it is not observed in InAs Nw based FETs; this could be likely ascribed to the different surface/electronic properties of the two materials. Of course, this model is very schematic both in estimating the nanowire interface shell extension and in neglecting the role of contacts, which would require a full 3D simulation and the inclusion of complicated band structure and semi-empirical parameters to reproduce the interface-related transport effects. On the other hand it gives a qualitative idea of a possible explanation for our current/detection curves.

In conclusion, we have reported the experimental demonstration of $\mathrm{THz}$ radiation detection in $\mathrm{InAs} / \mathrm{InSb}$ $\mathrm{Nw}$ based FETs. While the device responsivity is comparable to the one found in InAs based FETs, the presence of InSb offers new phenomenology and highlights the different device physics. This is evident in the peculiar trend of the photodetection curve, with a sign change in the video signal voltage, which has been qualitatively explained by the field asymmetries induced by a single lateral gate electrode. A. P. wishes to acknowledge funding from Regione Toscana through the POR project "Focus on competence" and Marie Curie Actions under REA grant agreement 298861 (NEMO).

${ }^{1}$ M. S. Vitiello, D. Coquillat, L. Viti, D. Ercolani, F. Teppe, A. Pitanti, F. Beltram, L. Sorba, W. Knap and A. Tredicucci, Nano Lett. 12, 96 (2012).

${ }^{2}$ M. S. Vitiello, L. Viti, L. Romeo, D. Ercolani, G. Scalari, J. Faist, F. Beltram, L. Sorba, and A. Tredicucci, Appl. Phys. Lett. 100, 241101 (2012).

${ }^{3}$ A. Pitanti, D. Ercolani, L. Sorba, S. Roddaro, F. Beltram, L. Nasi, G. Salviati and A. Tredicucci, Phys. Rev. X 1, 011006 (2011).

${ }^{4}$ H. A. Nilsson, P. Caroff, C. Thelander, E. Lind, O. Karlsrtöm and L. E. Wernersson, Appl. Phys. Lett. 96, 153505 (2010).

${ }^{5}$ I. Vurgaftman, R. Meyer and L.R. Ram-Mohan, J. Appl. Phys. 89, 5815 (2001).

${ }^{6}$ P. Caroff, J. B. Wagner, K. A. Dick, H. A. Nilsson, M. Jeppsson, K. Deppert, L. Samuelson, L. R. Wallenberg and L.-E. Wernersson, Small 4, 878 (2008).

${ }^{7}$ D. Ercolani, F. Rossi, A. Li, S. Roddaro, V. Grillo, G. Salviati, F. Beltram and L. Sorba, Nanotechnology 20, 505605 (2009).

${ }^{8}$ A. Pitanti, S. Roddaro, M. S. Vitiello and A. Tredicucci, J. Appl. Phys. 111, 064301 (2012).

${ }^{9}$ D. B. Suyatin, C. Thelander, M. T. Björk, I. Maximov and L. Samuelson, "Sulphur passivation for ohmic contact formation to InAs nanowires" Nanotechnology 18, 105307 (2007).

${ }^{10}$ W. Knap, M. Dyakonov, D. Coquillat, F. Teppe, N. Dyakonova, J. Łusakowski, K. Karpierz, M. Sakowicz, G. Valusis, D. Seli- 
uta, I. Kasalynas, A. El Fatimy, Y. M. Meziani and T. Otsuj, J. Infrared Milli. Terahz. Waves 30, 1319 (2009).

${ }^{11}$ M. A. Khayer and R. K. Lake, Electron Devices Meeting, 2008. IEDM 2008. IEEE International, 1 (2008).

${ }^{12}$ M. Sakowicz, M. B. Lifshits, A. Klimenko, F. Schuster, D. Coquillat, F. Teppe and W. Knap, J. Appl. Phys. 110, 054512 (2011).

${ }^{13} \mathrm{In}$ our particular case, the impedance is $60 \mathrm{M} \Omega$.
${ }^{14}$ S. Roddaro, A. Pescaglini, D. Ercolani, L. Sorba and F. Beltram, Nano Lett. 11, 1695 (2011)

${ }^{15}$ S. A. Dayeh, D. P. R. Aplin, X. Zhou, P. K. L. Yu, E. T. Yu and D. Wang, Small 3, 326 (2007).

${ }^{16}$ A. C. Ford, J. C. Ho, Y.-L. Chueh, Y.-C. Tseng, Z. Fan, J. Guo, J. Bokor and A. Javey, Nano Lett. 9, 260 (2009). 